\title{
THE AMERICAN ORTHOPSYCHIATRIC ASSOCIATION, INC.
}

\section{MEMBERSHIP LIST}

The symbol following the address indicates membership status: (C) Charter member; (F) Fellow; (H) Honorary. Numbers in parentheses indicate year of election to membership. The chief organization connection is followed by the mailing address, which may be either the home address or that of the organization.

Abbate, Grace McL., M.D., Bur. Child Guidance; 141 Joralemon St., Brooklyn 2, N. Y. (F) ('41)

Abbot, E. Stanley, M.D.; P.O. Box 119, Wayland, Mass. (F) ('29)

Ackerly, S. Spafford, M.D.; Mental Hyg. Clinic, 610 S. Floyd St., Louisville 2, Ky. (F) ('31)

Ackerman, Nathan W., M.D., Jewish Board of Guardians; 43 E. 78th St., New York 21 (F) ('39)

Adams, Walter A., M.D., Provident Hospital; 6011 Loomis Blvd., Chicago 36, Ill. (F) ('35)

Adamson, Eliza beth I., M.D.; Children's Court, 424 County Office Bldg., White Plains, N. Y. (F) ('42) Aldendorff, Herbert, M.D.; 320 West End Ave., New York 23 ('46)

Alexander, Franz, M.D.; Inst. for Psychoanalysis, 43 E. Ohio St., Chicago 11, Ill. (F) ('31)

Alexander, Vance T., M.D.; Child Guid. Clinic, 1122 N. Cascade Ave., Colo. Springs, Colo. ("46)

Allen, Cornelia H., Univ. of Buffalo, School Soc. Work; 195 Voorhees Ave., Buffalo 14, N. Y. (F) ('30)

Allen, Frederick H., M.D.; Child Guid. Clinic, 1711 Fitzwater St., Philadelphia 46, Pa. (F) ('26)

Allen, Sylvia, M.D., Univ. Kansas Medical College; 1010 Professional Bldg., Kansas City 6, Mo. ("46)

Alpern, Evelyn, M.D.; 545 Breckenridge St., Buffalo, N. Y. (F) ('33)

Alpert, Augusta, Ph.D., Mt. Sinai Hospital; 105 East 38th St., New York 16 ('40)

Altman, Charlotte H., Institute for Juvenile Research; 5524 S. Ellis Ave., Chicago 37, Ill. ('46)

Amster, Mrs. Fanny; 704 Highland Ave., Austin 21, Texas (F) ('39)

Anderson, Forrest N., M.D.; 14317 Huston St., Van Nuys, Calif. (F) ('29)

Anderson, Gladys L., Ph.D.; Child Guid. Clinic, 505 S. Fifth St., Champaign, Ill. ('45)

Anderson, Harolo H., Ph.D.; Dept. Psychology, Univ. of Illinois, Urbana, Ill. (F) ('39)

Anderson, Rose G., Ph.D.; Psychological Service Center, 522 Fifth Ave., New York 18 (F) ('37)

Anderson, V. V., M.D.; The Anderson School, Staatsburg-on-Hudson, N. Y. (F) (C)

Andrus, Ruth, Ph.D.; State Department of Education, Albany 1, N. Y. ('45)

Aptekar, Herbert H., Foster Home Bureau; 67-125 Dartmouth St., Forest Hills, N. Y. (F) ('39)

Arkin, Frances S., M.D.; 572 Park Ave., New York ('39)

Armstrong, Elizabeth W., Children's Center; 723 Seyburn Ave., Detroit, Mich. (F) ('40)

Arrington, Winifred W., N.Y.C. Comm. on Mental Hyg.; 621 W. 171 st St., New York 32 (F) ('30) Arthur, Grace, Ph.D.; 35 North Grotto, St. Paul 5, Minn. (F) (32)

Aтchley, Ruth H., St. Christopher's School; 51 Buena Vista Drive, Hastings-on-Hudson, N. Y. ('46) Aten, Eugene L., M.D., Child Guidance Clinic; 3511 Fairmount St., Dallas 4, Texas (F) ('38)

August, Harry E., M.D.; 26081 Hendrie Blvd., Huntington Woods, Mich. (F) ('33)

Barbee, Margaret; Sheltering Arms Children's Service, 124 E. 29th St., New York 16 ('33)

Barger, William C., M.D., Bur. Child Guidance; 30 Old Field Lane, Great Neck, L. I., N. Y. ("42) Barhash, A. Z, M.D.; 39 Madison Ave., Huntington, N. Y. (F) ('42)

Bartelme, Phyllis, Ph.D., U. S. Employment Service; 2350 Ward St., Berkeley 5, Calif. (F) ('29)

Bartmeier, Leo H., M.D., Henry Ford Hosp.; 57 Cloverly Rd., Grosse Pointe Farms 30, Mich. (F) ('29)

Baruch, Dorothy W., Ph.D.; 226 S. Gale Drive, Beverly Hills, Calif. (F) ('40)

Basamania, BetTy W., Judge Baker Guidance Center; 41 S. Russell St., Boston, Mass. ('46)

Baschnagel, Catherine; Mental Hyg. Soc. of Union County, 111 E. Front St., Plainfield, N. J. ('43)

Bassett, Clara; Mental Hygiene Clinic, 601 W. Lombard, Baltimore 1, Md. (F) ('28)

Beaghler, H. Edward, M.D.; 1332 N. Buena Vista, Apt. C, Burbank, Calif. ('43)

Beck, Samuel J., Ph.D.; Michael Reese Hospital, 29th St. \& Ellis Ave., Chicago 16, Ill. (F) (29)

Beckmann, J. William, M.D., Bureau of Child Guidance; 45 Prospect Place, New York 17 (F) ('41)

Beeley, Arthur L., Ph.D.; University of Utah, Salt Lake City 2, Utah (F) ('37)

Belcher, Esther L., Dept. Public Instruction; 535 Glenmore, Apt. 142A, East Lansing, Mich. ('40)

Bender, Lauretta, M.D.; Psychiatric Div., Bellevue Hosp., First Ave. \& 30th St., New York 16 (F) ('37)

Benjamin, Anne R., M.D.; 43 East Ohio St., Chicago 11, Ill. ('46)

Benton, Arthur L., Ph.D.; Student Personnel Bur., C.C.N.Y., Convent Ave. and 140th St., New York 31 (F) ('38)

Beres, David, M.D., Foster Home Bureau; 59 East 79th St., New York 21 ('44) 
Berlin, Mrs. Caroline J.; 5711 Pocusset St., Pittsburgh 17, Pa. ('32)

Berman, Leo, M.D.; 464 Beacon St., Boston 15, Mass. ('46)

Berman, StDneY, M.D.; Major, MC, Station Hospital, Bolling Field, Washington 20, D. C. ('43)

Bernard, Viola W., M.D., Bureau of Child Guidance; 930 Fifth Ave., New York 21 (F) ('42)

Berner, Frank, M.D., College of P. \& S., Columbia Univ.; 133 E. 58th St., New York 22 ('41)

Bernstein, Nathaniel, M.D.; Bur. Child Guidance, 1550 Crotona Park East, Bronx 60, N. Y. ('46)

Binder, Harold J., M.D.; Capt., MC, Lawson General Hosp., Atlanta, Ga. ('42)

Blanchard, Myron B.; National Jewish Welfare Bd., Rm. 805, 55 W. 42nd St., New York 18 (F) ('34)

Blanchard, Phyllis, Ph.D.; Child Guid. Clinic, 1711 Fitzwater St., Philadelphia 46, Pa. (F) ('30)

Blank, H. RoBert, M.D.; Capt., MC, Mason General Hospital, Brentwood, N. Y. ('46)

Blau, Abram, M.D.; 47 East 88th St., New York 28 (F) ('39)

Blos, Perer, Ph.D., Personnel Bureau, Brooklyn College; 820 Park Ave., New York 21 ('44)

Bone, Harry, Ph.D.; 400 West 119th St., New York 27 ('43)

Bradley, Charles, M.D.; Emma Pendleton Bradley Home, Riverside 15, R. I. (F) ('36)

Branham, V. C., M.D.; Institute for Defective Delinquents, Woodbourne, N. Y. ('42)

Bremner, Elizabeth, A., M.D.; Conn. Bureau Mental Hygiene, 1179 Main St., Hartford 3, Conn. ('46)

Brenman, Margaret, Ph.D.; The Menninger Clinic, Topeka, Kansas ('44)

Brickner, Ruth P., M.D.; 4926 Goodridge Ave., New York 63 ('34)

Bridgman, Olga, M.D., Dept. Public Health; 1380 Monterey Blvd., San Francisco 16, Calif. (F) ('25)

Bridgman, Ralph P.; Hampton Institute, Hampton, Va. (F) ('31)

Brinker, Dorothy L., Martha H. Beeman Child Guid. Clinic; Jefferson \& 3rd St., Niagara Falls, N. Y. (F) ('32)

Broadwin, IsRa T., M.D., C.C.N.Y., School of Education; 116 W. 59th St., New York 19 (F) ('30)

Brockbank, Thomas W., M.D.; Catholic Charities Guid. Inst., 133 E. 58th St., New York 22 (F) ('33)

Bromberg, Norbert, M.D.; 39-32 47th St., Long Island City 4, N.Y. ('43)

Bromberg, Walter M., M.D.; 140. No. Virginia St., Reno, Nevada (F) ("41)

Bronner, Augusta F., Ph.D.; Judge Baker Guid. Center, 38 Beacon St., Boston 8, Mass. (F) ('25)

Brown, Muriel W., Ph.D.; U. S. Office of Education, Washington 25, D. C. (F) ('30)

Bumstead, Kate S.; 101 East 36th St., Kansas City 2, Mo. ('44)

Burling, Temple, M.D.; Child Guidance Clinic, 100 N. Main St., Providence 3, R. I. (F) ('33)

Burton, Aileen C.; 347 East 18th St., New York 3, ('42)

Buxbaum, Edith, Ph.D.; 210 East 68th St., New York 21 ('43)

Buxton, Rex E., M.D., Washington Inst. Ment. Hyg.; 5202 Western Ave., Chevy Chase 15, Md. (F) ('40)

Cameron, Eugenia S., M.D., State Board of Health; 3507 Sunset Dr., Shorewaod Hills, Madison 5, Wis. (F) ("29)

Campbell, Douglas G., M.D.; 160 Palo Alto Ave., San Francisco 14, Calif. ('40)

Carroll, Clara; Bureau Child Guid., Queens Unit, 166th St. \& 65th Ave., Flushing, N. Y. ('42)

Carter, Jerry W., JR., Ph.D.; Child Guid. Center, 3422 E. Douglas, Wichita 8, Kansas ('41)

CARY, Mrs. Richard; R.D. River Road, Lewiston, N. Y. (F) ('31)

Cassino, Frank A., M.D.; Capt., MC, Walter Reed General Hosp., Washington, D. C. ('43)

Challman, S. Alan, M.D.; 1945 Medical Arts Bldg., Minneapolis 2, Minn. (F) ('35)

Chamberlate, Herbert E., M.D.; P.O. Box 933, Sacramento, Calif. (F) ('27)

Chapman, Ross McC., M.D.; Sheppard \& Enoch Pratt Hospital, Towson 4, Md. (F) ('28)

Chassell, Joseph, M.D.; Bennington College, Bennington, Vt. (F) ('38)

Chennell, Eireen A., M.D.; Military Hospital (Women), Northampton, England ('44)

Childers, Asher T., M.D., Central Mental Hygiene Clinic; 1721 Berkley Ave., Cincinnati 29, Ohio (F) ('31)

ChornyaK, John, M.D.; Box 1866, Chicago 90, Ill. (F) ('38)

CHov, LI-ChIv; Department of Sociology, Ginling College, Chengtu, West China ('36)

Clark, Josephine E.; Guidance Center, 1737 Prytania St., New Orleans 13, La. ('42)

Clark, Mary Augusta; Bedford, N. Y. (F) ('30)

Clemence, Esther H., Institute for Juvenile Research; 1037 Cherry St., Winnetka, Ill. ('45)

Clifton, Eleanor, Community Service Society; 15 West 11 th St., New York 11 ('27)

Clothier, Florence, M.D.; New England Home for Little Wanderers, 161 S. Huntington Ave., Boston 30 , Mass. (F) ('42)

Cochran, Mrs. Mary Lue; Health Department, 472 Indiana Ave., Washington 1, D. C. ('31)

Cochill, Harvie deJ., M.D.; Chn's Memorial Clinic, 1001 E. Clay St., Richmond 19, Va. (F) ('28)

Cohen, Anna, N. Y. Association for Jewish Children; 80 Aycrigg Ave., Passaic, N. J. ('41) 
Coleman, Jules V., M.D.; 4200 E. 9th Ave., Denver 7, Colo. ('39)

Conn, Jacoв H., M.D., Johns Hopkins Hospital; 2325 Eutaw Place, Baltimore 17, Md. (F) ('40)

Cook, Stuart W., Ph.D.; Comm. on Community Interrelations, 212 W. 50th St., New York 19 ('43)

Cooper, Olive A., M.D., Child Guidance Clinic; 160 Maple St., Springfield 5, Mass. (F) ('41)

Corman, Bertha, Jewish Children's Bureau; 5519 Drexel Ave., Chicago 37, Ill. ('42)

Corson, Harold F., M.D.; c/o Box 145, Stockbridge, Mass. ('31)

Cottington, Frances, M.D.; 40 East 61 st St., New York 21 ('41)

Couch, Mildred Warden, M.D.; Cromwell Hall, Cromwell, Conn. ('31)

Crampton, Jessie E.; Child Guidance Center, 201 Montague St., Brooklyn 2, N. Y. (F) ('41)

Crane, Harry W., Ph.D.; Drawer 809, Chapel Hill, N. C. ('37)

Crothers, Bronson, M.D.; Children's Hospital, 300 Longwood Ave., Boston 15, Mass. (F) ('41)

CRowell, Sarah E.; El Alamo Viejo, RFD \#1, Albuquerque, New Mexico ('36)

Crutcher, Hester B.; Dept. of Mental Hygiene, State Office Bldg., Albany 1, N. Y. (F) ('41)

Crutcher, Roberta, M.D., Pasadena Ch. Guid. Clinic; 827 S. Hobart Blvd., Los Angeles 5, Calif. ('45)

Cunningham, J. M., M.D.; Bureau Mental Hygiene, State Dept. Health, Hartford 6, Conn. (F) ('33)

Cunningham, Mrs. Lucretia B., Hartford Council of Social Agencies; 315 Ridgewood Rd., West Hartford, Conn. ('46)

Curtis, WilliaM B., M.D.; Psychiatric Service in Community, 195 Church St., New Haven, Conn. (F) ('41)

Davidofr, Eugene, M.D.; Craig Colony, Sonyea, N. Y. ('42)

Davis, Anne E., Institute for Juvenile Research; 5532 Kenwood Ave., Chicago 37, Ill. (F) ('41)

Davis, Margaret S., Welfare Council; 251 Metcalf St., Ottawa, Canada (F) ('34)

Dawley, Almena; Child Guidance Clinic, 1711 Fitzwater St., Philadelphia 46, Pa. (F) ('31)

DE Berry, ElletT M., M.D.; Sonora, Texas (F) ('29)

Despres, Marian A., Ph.D.; 1220 East 56th St., Chicago 37, Ill. ('39)

Deursch, Regina, Bureau of Child Guidance; 2010 Grand Ave., New York 53 ('43)

Diamond, Bernard L., M.D., Langley-Porter Clinic; 20 Romolo Place, San Francisco 11, Calif. ('46)

Dicks Grace H.; A.R.C., Military \& Naval Welf. Serv., 230 Spring St., N.W., Atlanta 3, Ga. (F) ('37)

Dimmick, Graham B., Ph.D.; Child Guidance Service, 217 N. Upper St., Lexington 15, Ky. ('43)

Dolan, Dorothea L., Veterans Administration, Soc. Serv. Div., Branch 10, Dallas 1, Texas ('42)

Doll, Edgar A., Ph.D.; The Training School, Vineland, N. J. (F) ('25)

Dombey, Edith H.; Juvenile Court, East 22nd St. \& Cedar Ave., Cleveland 15, Ohio ('34)

Dominquez, Kathrys E.; Children's Service Center, 335 S. Franklin St., Wilkes-Barre, Pa. ('45)

Doniger, Simon, Ph.D.; Jewish Child Guidance Bureau, 682 High St., Newark 2, N. J. ('41)

Doody, William M., M.D., Catholic Charities of N. Y.; 19 Bentley Ave., Jersey City 4, N. J. ('35)

Dorsey, John M., M.D.; The Children's Center, 3742 Brush St., Detroit 2, Mich. ('46)

Dummer, Mrs. W. F.; 679 N. Michigan Ave., Chicago 11, Ill. (H) ('26)

Dunbar, Flanders, M.D., Presbyterian Hospital; 3 East 69th St., New York 21 ('43)

Durfee, Marion B., M.D.; School Guidance Center, 508 College St., Los Angeles 12, Calif. ('43)

Durkin, Helen E., Ph.D., New Rochelle Child Guid. Clinic; 7 Fairview Rd., Scarsdale, N. Y. ('42)

Dybwad, Gunnar; State Dept. Social Welfare, 230 N. Grand Ave., Lansing 4, Mich. ('42)

Ebaugh, Frankim G., M.D.; Colo. Psychopathic Hospital, 4200 E. 9th Ave., Denver, Colo. (F) ('29)

Eisler, Edwin R., M.D.; Inst. for Psychoanalysis, 43 E. Ohio St., Chicago 11, 111. (F) ('29)

Eisner, Eugene A., M.D.; 161 East 91 st St., New York ('46)

ELKIND, HENRY B., M.D., (Address unavailable) (F) ('28)

Emery, E. Van Norman, M.D.; 4668 Pershing Ave., St. Louis 8, Mo. (F) ('31)

Englander, Charles, M.D.; 41 Hillside Ave., Newark 8, N. J. (F) ('31)

Ephron, Harmon S., M.D.; 295 Central Park West, New York 24 (F) ('38)

Epstein, Morris D., M.D.; 49 East 96th St., New York 28 ('41)

Errkson, ERIK H., Inst. Child Welf,, Univ. of California; 25 Hacienda Rd., Orinda, Calif. (F) ('42)

Escalona, Mrs. Sibylle; The Menninger Clinic, Topeka, Kansas ("46)

Estes, Stanley G., Ph.D., Northeastern University; 60 Pinckney St., Boston 14, Mass. ('41)

Evans, Chester E., Ph.D.; Labor Economics Section, General Motors Corp., 3044 W. Grand Blvd., Detroit 2, Mich. ('45)

Ewerhardt, PaUL J., M.D., Chn's. Hosp.; 1028 Connecticut Ave., N.W., Washington, D. C. (F) ('30)

Fabian, Abraham A., M.D., Brooklyn Child Guidance Centre; 15 E. 10th St., New York 3 ('45)

Falstein, Eugene I., M.D., Michael Reese Hosp.; 185 N. Wabash Ave., Chicago, Ill. ('39) 
Feder, Leah, Ph.D.; Family Society, 400 Community Fund Bldg., Pittsburgh, Pa. (F) ('39)

Fenlason, Mrs. L. S.; University of Minnesota, 8 Jones Hall, Minneapolis 14, Minn. (F) ('37)

Fenton, Norman, Ph.D.; P.O. Box 18, San Quentin, Calif. ('37)

Ferguson, W. G., M.D.; Martha H. Beeman Clinic, 650 Memorial Parkway, Niagara Falls, N. Y. (F) ('30)

Field, Mrs. Minna, Westchester County Council of Soc. Agencies; 155 W. 20th St., New York 11 ('46)

Finley, Malcolm H., M.D., Guidance Serv,, Girls H.S.; 290 Brentwood Ave., San Francisco 16, Calif. (F) ('37)

Firor, Marion, M.D.; Child Guidance Clinic, 1325 W. Adams Blvd., Los Angeles 7, Calif. ('42)

Fischer, Liselotte K., Ph.D.; Hartley-Salmon Clinic, 79 Farmington Ave., Hartford, Conn. ('46)

Fishback, Dora, M.D.; 43 E. Ohio St., Chicago 11, Ill. ('44)

Fitzherbert, Florence, Community Service Society; 130 E. 67th St., New York ('43)

Fitz-Simons, Marian J., Ph.D., Grosse Pointe Bd. of Ed.; 1130 Parker Ave., Detroit 14, Mich. (F) ('37)

Fleming, Alice M.R.; Lt. W-V(S)(H)USNR, Naval Hospital, Newport, R. I. ('42)

Font, Marion McKenzie; Tulane University Med. School, 1430 Tulane Ave., New Orleans, La. ('41)

Foster, Roberta; Akron Child Guidance Center, 312 Locust St., Akron 3, Ohio ('41)

Foster Sybil; Adoption Service Bureau, Room 501, 1001 Huron Rd., Cleveland 15, Ohio (F) ('30)

Foxe, Arthur N., M.D.; 25 West 54th St., New York 19 ('41)

Francis, Mrs. Blythe W.; Family Welf. Assoc., 126 W. 3rd St., Los Angeles 13, Calif. (F) ('37)

Frank, Lawrence K.; 72 Perry St., New York 14 ('F) ('32)

Frankl, Mrs. Anni W.; Child Guidance Clinic, 1020 McGee St., Kansas City, Mo. ('42)

Frankl, George, M.D.; Child Guidance Clinic, 1020 McGee St., Kansas City, Mo. ('44)

Frankley, Greta, M.D.; 350 Central Park West, New York 25, ('39)

French, Lois Meredith; 98 Alexander Ave., Nutley, N. J. (F) ('33)

Friend, Maurice R., M.D., Capt., MC, Vaughan General Hosp., Hines, 1ll.; c/o Dr. Emanuel

London, 1901 Ave. N, Brooklyn 30, N. Y. ('43)

Fries, Margaret E., M.D.; 21 West 86th St., New York 24 (F) ('36)

Fry, Clements C., M.D.; 109 College St., New Haven, Conn. (F) ('31)

Fulder, Earl W., M.D., Northern N. J. Ment. Hyg. Clinics; 8 Mill Rd., Morris Plains, N. J. (F) ('31)

Gabell, Marcene P.; Box 216, South Yarmouth, Mass. ('42)

Gardner, George E., Comdr., MC, USNR, Washington 25, D. C.; c/o 7 Trapelo Rd., Belmont, Mass. (F) ('41)

Garrett, Annette; Smith College School for Social Work, 8 College Hall, Northampton, Mass. (F) ('31)

Gartland, Ruth, School of Applied Soc. Sciences, Univ. of Pittsburgh; 1033 Devonshire Rd., Pittsburgh 13, $\mathrm{Pa}$. (F) ('42)

Gaw, EmIR A., M.D.; 1190 Shattuck Ave., Berkeley 7, Calif. ('43)

Geleerd, Elisabeth R., M.D.; 44 East 80th St., New York 21 ('43)

Gerard, Margaret W., M.D.; 5744 Harper Ave., Chicago 37, Ill. (F) ('31)

Gessell, Arnold, M.D.; Clinic Child Develop., 14 Davenport Ave., New Haven 11, Conn. (F) ('31)

Gilbert, Lovis Jay, M.D., Bureau of Child Guidance; 235 E. 22nd St., New York ('44)

Gildea, Margaret C.-L., M.D., Washington University School of Medicine; 4445 Lindell Blvd., St. Louis 8, Mo. ('44)

Gilmore, John V., Harvard-Boston University Extension; 6 Gorham St., Cambridge, Mass. ('46)

Ginsburg, Mrs. Ethel L.; 12 East 97 th St., New York 29 (F) ('39)

Ginsburg, Sol W., M.D., Mt. Sinai Hospital; 12 East 97 th St., New York 29 ('45)

Gitelson, Maxwell M.D.; 55 East Washington St., Chicago, Ill. (F) ('36)

Glatzer, Henriette T., Brooklyn Child Guid. Center; 30 Disbrow Lane, New Rochelle, N. Y. ('42)

Glauber, Helen M.; 829 Park Ave., New York 21 ('41)

Gluckman, Martha H., Ph.D.; 134 Haven Ave., New York 34 ('33)

Goldberg, Milton, M.D.; 1321 West Wood Blvd., Los Angeles 24, Calif. ('45)

Goldensohn, Leon N., M.D.; Major, MC (0-503397) Int. Security Det., Int'l Military Tribunal, APO 124-A, c/o Postmaster, New York ('42)

Goldfarb, William, Ph.D., Foster Home Bureau; 720 Riverside Dr., New York ('45)

Goldman, Julia, N. Y. Assoc. for Jewish Children; 1230 Park Ave., New York (F) ('33)

Goldman, Nathan; 9825 S. Seeley Ave., Chicago 43, Ill. ('39)

Goodman, Soll, M.D.; Psychiatric Institute, 722 West 168th St., New York 32 ('44)

Gould, Miriam C.; 333 East 30th St., New York 16 ('33)

Green, Rose; School of Social Work, University of Southern California, University Pk., Los Angeles, Calif. (F) ('38) 
Greenacre, Phyllis, M.D., New York Hospital; 970 Park Ave., New York 28 (F) ('43)

Griffith, Mrs. Carleton; 1050 Logan St., Denver 3, Colo. ('42)

Grossman, Royal G., M.D.; 1423 Arthur Ave., Lakewood, Ohio ('28)

Grossmann, Grace; Nat'l Council Jewish Women, 285 Schermerhorn St., Brooklyn 17, N. Y. (F) ('31)

Groves, ERNest R.; University of North Carolina, Box 929, Chapel Hill, N. C. (F) ('41)

Hacker, Fredericx J., M.D.; 247 S. Beverly Drive, Beverly Hills, Calif. ('45)

Hagan, Margaret W., A.R.C., St. Elizabeth's Hosp.; 305 Tenth St., N.E., Washington, D. C. (F) ('36)

Hagman, Elmer R., Ph.D.; 83 Meadowbrook Road, West Hartford 7, Conn. (F) ('33)

Halpern, Florence, Bellevue Hospital, Psychiatric Division; 222 E. 82nd St., New York 28 ('44)

Hamilton, E. Louise, Ph.D.; 1828 W. Tioga St., Philadelphia 40, Pa. ('39)

Hankins, Dorothy; Child Guidance Clinic, 1711 Fitzwater St., Philadelphia 46, Pa. (F) ('40)

HannetT, FRances; M.D., Univ. of Chicago Health Serv.; 5490 S. Shore Drive, Chicago 15, Ill. (F) ('39)

Hanson, Harold B., M.D., U. S. Children's Bureau; 1995 Bayard Ave., St. Paul 5, Minn. (F) ('39)

Hardcastle, Dorothy H., Mental Health Emergency Comm.; 2 Lyndhurst Rd., Hampstead, London N.W. 3, England ('41)

Hardcastle, Douglas N., M.D., Ministry of Health; 48 Warwick Rd., Bishops Stortford, Hertfordshire, England ('34)

Harle, Marjorie; Child Guidance Clinic, 21 Catherine St., Worcester 5, Mass. ('44)

Harms, Herbert E., M.D.; 566 Radnor Road, Oakland 6, Calif. ("41)

Harris, June; Bureau Child Guidance, 228 E. 57th St., New York 22 ('42)

Harrison, Frances; Norristown State Hospital, 1720 Markley St., Norristown, Pa. ('32)

Hartwell, S. W., M.D.; Grand Valley Children's Center, 124 Michigan St., N.E., Grand Rapids 3, Mich. (F) ('29)

Healy, William, M.D.; Judge Baker Guidance Center, 38 Beacon St., Boston 8, Mass. (F) (C)

Heath, Esther, Pasadena Child Guidance Clinic; 107 Grace Terrace, Pasadena 2, Calif. ('45)

Heldt, Thomas J., M.D.; Henry Ford Hospital, Detroit 2, Mich. ('29)

Helgesson, UNo H., M.D.; (address unavailable) ('31)

Hennessy, Maurice A. R., M.D.; 10515 Carnegie Ave., Cleveland 6, Ohio ('31)

Hen rich, Mildred R.; Jewish Board of Guardians, 228 East 19th St., New York 3 ('46)

Henry, Jules, Ph.D., Office of Inter-American Affairs; 3640 Gunston Rd., Alexandria, Va, ('43)

Herskovitz, Herbert, M.D., Berks County Guidance Institute; 245 N. 5th St., Reading, Pa. ('44)

Hertz, Marguerite E., Ph.D., Western Res. Univ.; 2835 Drummond Rd., Cleveland 20, Ohio (F) ('42)

Hertzman, Jack, M.D., Board of Education; 19 Garfield Place, Cincinnati 2, Ohio ('39)

Hicks, Helen R.; 5400 Whittaker Road, Ypsilanti, Mich. ('41)

Hrll, Julia Ford, M.D.; Child Guidance Center, 3604 Victoria St., Pittsburgh 13, Pa. (F) ('35)

Hill, Lewis B., M.D., Smith College Sch. for Soc. Work; 10 W. Madison St., Baltimore 1, Md. (F) ('38)

Hrscks, C. M., M.D.; Canadian National Comm. for Mental Hygiene, 111 St. George St., Toronto, Canada (F) ('33)

Hincks, Eliza beth M., Ph.D.; 51 Brattle St., Cambridge 38, Mass. ('32)

Hirsch, Jeannette S.; 241 Central Park West, New York 24 ('42)

Hochreiter, Mrs. F. C.; 601 Starin Ave., Kenmore 17, N. Y. ('41)

Holmer, Paul, M.D.; Box 344, Kentfield, Calif. (F) ('34)

Holt, William L., JR., M.D.; Westborough State Hospital, Westborough, Mass. ('40)

Holzberg, Jules D., Lt., MAC; Mason General Hospital, Brentwood, N. Y. ('45)

Horton, Charles B., M.D.; 12 East 86th St., New York 28 (F) ('33)

Houtz, Mrs. Philtp, Jewish Board of Guardians; 124 West 79 th St., New York ('44)

Humphreys, Edward J., M.D.; Bureau Mental Hygiene, State Office Bldg., Columbus 16, Ohio ('45)

Irons, Lucia; Calvert Court, Apt. D8, Baltimore 18, Md. ('39)

Ittleson, Mrs. Blanche; 965 Fifth Ave., New York 21 (H) ('40)

Jackson, Edrth B., M.D.; Dept. of Pediatrics, Yale University Medical School, 789 Howard Ave., New Haven 4, Conn. (F) ('38)

Jackson, James D.; 27A North Dean Ave., Trenton 8, N. J. ('30)

JaEnike, R. C. A., M.D.; Child Guidance Clinic, Strong Memorial Hospital, Rochester 7, N. Y. ('33)

January, Mildred H., M.D., Board of Education; 111 Gillett St., Hartford 5, Conn. ('44)

Janvier, Carmelite, Div. Special Serv., Pub. Schools; 3307 Prytania St., New Orleans 15, La. (F) ('35)

Jefrerson, R. A., M.D., Marquette Univ. Sch. of Med.; 208 E. Wisconsin Ave., Milwaukee 2, Wis. ('33)

Jenkins, R. L., M.D.; Champaign-Urbana Pub. Health Dist., 1306 W. Stoughton, Urbana, Ill. 
Jensen, Reynold A., M.D., Univ. of Minnesota Hosp.; 2412 Seabury Ave., Minneapolis 6, Minn. ("46) Jessner, Lucie, M.D., Habit Clinic for Child Guidance; 107. Garden St., Cambridge 38, Mass. ("45) Johnson, Adelaide M., M.D., Inst. for Psychoanalysis; 5807 Dorchester Ave., Chicago 37, Ill. (F) ('41) Johnson, Eleanor H., Ph.D.; 351 Farmington Ave., Hartford 5, Conn. (F) (26)

Johnson, George S., M.D.; Stanford University Hospital, Clay \& Webster Sts., San Francisco 15, Calif. (F) ('30)

Jones, Harold E., Ph.D., Inst. Child Welf., University of California; 2683 Shasta Rd., Berkeley 8, Calif. (F) ('38)

Joslyn, June J.; Oregon Mental Hygiene Society, 318 Platt Bldg., Portland 5, Oregon (F) ('34)

Judxins, Barbara E.; Hartley-Saimon Clinic, 79 Farmington Ave., Hartford, Conn. ('46)

Karpman, Benjamin, M.D.; St. Elizabeth's Hospital, Washington, D. C. (F) ('39)

Kaufman, S. Harvard, M.D.; 211 Alaska Bldg., 2nd \& Cherry, Seattle 4, Wash. ('44)

Kawin, Erhel, Glencoe Public Schools; 1725 E. 53rd St., Chicago 15, Ill. (F) ('32)

Keiser, Sylvan, M.D., Bellevue Hospital; 941 Park Ave., New York 28 ('43)

Kemble, Robert P., M.D.; Address not available (F) ("40)

Kenworthy, Marion E., M.D.; 1035 Fifth Ave., New York 28 (F) ('24)

Kimball, Everett, Ph.D; College Hall 8, Smith College, Northampton, Mass. (F) ('30)

Kino, Anna, Austen Riggs Foundation, Inc.; Yale Hill, Stockbridge, Mass. ('42)

Kirkpatrick, M. E., M.D.; Guidance Center, 1737 Prytania St., New Orleans 13, La. (F) ('31)

KLein, Emanuel, M.D., Bureau Child Guidance; 47 E. 88th St., New York (F) ('40)

KLeinschmidt, SARA; Bureau of Crippled Children, Board of Health, Honolulu 1, T. H. ('40)

Knapp, Irving J., M.D.; New York State School for Boys, Warwick, N. Y. ('37)

Koch, Eleanor S., M.D.; 1640 Pine St., Philadelphia, Pa. ('42)

Kovarsky, Marcel; 6327 Alamo Ave., Clayton 5, Mo. (F) ('31)

Kris, Ernst, Ph.D., New School for Social Research; 135 Central Park West, New York 23 ('44)

Krug-Brady, Othilda, M.D.; Central Mental Hygiene Clinic, General Hospital, Cincinnati, Ohio ("44)

Krugman, Morris, Ph.D.; Bureau of Child Guidance, 228 E. 57 th St., New York 22 (F) ('39)

Kundert, Eliza beth, M.D.; Mental Hygiene Clinics, 120 State St., Montpelier, Vt. ('44)

LaBine, Altred C., M.D.; 8-259 General Motors Bldg., Detroit 2, Mich. ('28)

LaMar, Norvelle C., M.D., Payne Whitney Clinic; 149 E. 73rd St., New York 21 (F) (30)

Lander, Joseph, M.D.; Mercantile Library Bldg., Suite 1009, Cincinnati 2, Ohio ('40)

Landis, Edward E., M.D; Dept. Psychiatry, General Hosp., 323 E. Chestnut S., Louisville 2, Ky. ("42)

Landis, Marjorie R., Lehigh Valley Guidance Clinic; 713 Eighth Ave., Bethlehem, Pa. ("42)

Lanford, William S., M.D.; Pediatric Psychiatric Clinic, Babies Hospital, Broadway \& 167th St., New York 32, (F) ('36)

Langner, Helen P., M.D., New York Hospital; 40 East 61st St., New York 21 (F) ('29)

Larson, John A., M.D.; State Hospital, Las Vegas, New Mexico ('31)

Lavghead, Mary B., Fordham Univ. School Soc. Serv.; 44 Gramercy Pk. No., New York 10 (F) ('30)

LaVAllee, J. C. E., M.D., Catholic Guidance Clinic; 2123 Luray Ave., Cincinnati 6, Ohio ('39)

Lawton, George, Ph.D.; Old Age Counselling Service, 41 W. 82nd St., New York 24 (F) ('35)

Layman, Mary H., M.D., Stanford Medical School; 2156 Pacific Ave., San Francisco 15, Calif, ('42)

Ledwith, Mrs. W. M.; Child Guidance Center, 3604 Victoria St., Pittsburgh 13, Pa. ('45)

Leonard, Christina M., M.D., Fordham Univ. School of Social Service; 44 Gramercy Pk. No., New York $10(\mathrm{~F})(24)$

Leonard, Shirlex; Bureau of Child Guidance, 228 East 57th St., New York 22 (F) ('31)

Levey, Harry B., M.D.; Institute for Psychoanalysis, 43 East Ohio St., Chicago 11, Ill. (F) ('38)

Levi, Verna, Ph.D., Worcester Child Guidance Clinic; Box 57, State Hospital, Worcester 1, Mass. ('46)

Levine, Maurice, M.D., Univ. Cincinnati Med. School; 984 Lenox Place, Cincinnati 29, Ohio (F) ('41)

Levy, David M., M.D.; 300 Park Ave., New York (F) (C)

Lewin, Bertram D., M.D.; 32 East 64th St., New York 21 (F) (31)

Lewis, Margaret, Associated Charities, Cleveland; Park Lane, Chagrin Falls, Ohio (F) ('35)

Lewis, Murray D., M.D.; Capt., MC, Hdq. ASFTC, Ft. Lewis, Wash. ('42)

Libbey, Betsey; Family Society, 311 S. Juniper St., Philadelphia 7, Pa. ('31)

Lion, ERnest G., M.D., Stanford Univ. School of Medicine; 490 Post St., San Francisco 2, Calif. ('40)

Lippman, H. S., M.D.; Amherst H. Wilder Child Guid. Clinic, 279 Rice St., St. Paul 2, Minn. (F) ('28)

Liss, Edward, M.D., New York School for Social Research; 130 E. 39th St., New York 16 (F) ('32)

Lithauer, Donah B., N. Y. Association for Jewish Children; 150 W. 95th St., New York 25 ('32) 
Little, Harry M., M.D.; Child Guidance Center, 3604 Victoria St., Pittsburgh 13, Pa. (F) ('35) Lloxd, Ruth; 43 Linnaean St., Cambridge 38, Mass. ('43)

Lloyd, Wilma, Child Development Center, Children's Hospital of East Bay; 6100 Snake Rd., Oakland 11, Calif. (F) ('31)

Loewald, Hans W., M.D.; Ment. Hyg. Soc. of Maryland, 601 W. Lombard, Baltimore 1, Md. ('43)

Lono, Alroy S., Bureau of Child Guidance; 75 St. Nicholas Place, Apt. 2K, New York ("42)

Lorand, Sandor, M.D., Psychoanalytic Institute; 40 Central Park So., New York 19 (F) ('33)

LotT, George M., M.D., Suffolk County Dept. of Health; Box 66, Setauket, L. I., N. Y. ('33)

Lourie, Reginald S., M.D.; Guidance Center, 31 Gibbs St., Rochester 4, N. Y. ('41)

Lowrey, Lawson G., M.D., Columbia University; 25 W. 54th St., New York 19 (F) (C)

Lowry, Fern; N. Y. School of Social Work, 122 East 22nd St., New York 10 (F) ('37)

Lucas, Leon, Jewish Child Guid. Bureau, Newark; 2110 Bronx Park E., Bronx 60, N. Y. ('44)

Luckey, Bertha M., Ph.D.; Board of Education, 6th \& Rockwell, Cleveland 14, Ohio (F) ('31)

Luehrs, Lesie E., M.D.; 14 Washington Square North, New York 11 ('29)

Lurie, Lours A., M.D., Child Guidance Home; 19 Garfield Place, Cincinnati 2, Ohio (F) (26)

Lurie, Olga Rubinow, Ph.D.; 742 Halstead Ave., Mamaroneck, N. Y. (F) ('39)

Lyman, Richard, M.D.; Duke University, Durham, N. C. (F) ('34)

McAlpin, Katharine B.; Child Guidance Clinic, 1325 W. Adams Blvd., Los Angeles 7, Calif. ("44)

McBee, Marian; N.Y.C. Committee on Mental Hygiene, 105 E. 22nd St., New York 10 (F) (39)

McClure, Mrs, R. H.; 1036 Murrayhill Ave., Pittsburgh 17, Pa. (F) ('31)

McCord, Clinton P., M.D.; 54 Willett St., Albany 6, N. Y. (F) (26)

McLean, Helen V., M.D.; Institute for Psychoanalysis, 43 E. Ohio St., Chicago 11, 11l. (F) ('40)

McNiel, Edwin E., M.D.; 3875 Wilshire Blvd., Los Angeles 5, Calif. ('37)

MacDonald, Martha W., M.D.; Bureau Mental Hygiene, Queens Hospital, Honolulu, T. H. (F) ('33)

MacDougald, Eliza beth, M. D.; 1205 Scott Ave., Winnetka, Ill. ('44)

MacFarlane, Jean W., Ph.D.; 1220 Oxford St, Berkeley 7, Calif. (F) ('30)

Maeder, LeRoy M. A., M.D., Psychoanaly tic Inst.; 206 S. 13th St., Phila. 7, Pa. (F) ('29)

Magiera, Estelle A., M.D.; Child Guidance Clinic, 704 Standard Life Bldg., Jackson 27, Miss. ('45)

Mahaffy, Mrs. J. H., Family Welfare Assoc.; 521 6th St., S.E., Minneapolis, Minn. ('43)

Mahler, Margaret S., M.D.; 350 Central Park West, New York 25 ('45)

Malcove, Lillian, M.D., Psychoanaly tic Institute; 245 East 72nd St., New York ('31)

Mann, Edna B., Bureau of Child Guidance; 215 West 98th St., New York 25 ('42)

Markey, Oscar B., M.D.; 2923 Brighton Road, Shaker Heights 20, Ohio (F) ('28)

Marquis, Dorothy P., Ph.D.; University of Michigan, Dept. Psychology, Ann Arbor, Mich. ("44)

Marshall, Berry C., M.D.; 419 Hawthorn Road, Baltimore 10, Md. ('33)

Marshall, Helen; Dept. of Psychology, Stanford University, Calif. ('34)

Martin, Alexander R., M.D.; 24 Gramercy Park, New York 3 (F) ('42)

Martin, A. Leila; Psychological Services, Bd. of Ed., 13 Fitzhugh St. S., Rochester 4, N. Y. (F) (26)

Mathews, Julia; Child Guidance Clinic, 1325 W. Adams Blvd., Los Angeles 7, Calif. ('44)

Mathews, W. Mason, Ph.D.; Bureau Mental Hygiene, Board of Health, Honolulu, T. H. ('40)

Mayer, Edward E., M.D., University of Pittsburgh; 3401 Fifth Ave., Pittsburgh, Pa. ("34)

Mead, Margaret, Ph.D.; American Museum of Natural History, 15 W. 77 th St., New York 24 ('45)

Mexeel, H. Scudder, Ph.D.; 328-B Sterling Hall, University of Wisconsin, Madison 6, Wis. ('45)

Meldor, Ruth; Mental Hygiene Clinic, 610 S. Floyd St., Louisville 2, Ky. (F) ('39)

Meltzer, H., Ph.D., Psychological Serv. Center; 4510 Maryland Ave., St. Louis 8, Mo. (F) ('31)

Menaker, Esther, Ph.D.; 20 West 77 th St., New York 24 ('39)

Menger, Clara; Child Guidance Bureau, 10th \& Gaty, East St. Louis, Ill. ('43)

Menninger, Karl A., M.D.; The Menninger Clinic, Topeka, Kansas (F) (C)

Menninger, William C., M.D.; Brig. Gen., MC, The Menninger Clinic, Topeka, Kansas (F) ('35)

MeYer, Adolph, M.D.; 4305 Rugby Road, Baltimore 10, Md. (F) ('24)

Michaels, Joseph J., M.D.; 82 Marlboro St., Boston, Mass. (F) ('38)

Mille R, Joseph S. A., M.D.; Rockland State Hospital, Orangeburg, N. Y. ('41)

Mrtlet, John A. P., M.D., Presbyterian Hospital; 563 Park Ave., New York 21 ('45)

Mirviss, Sophia, M.D., Mt. Zion Hospital; 2441 Vallejo, San Francisco 23, Calif. ('44)

Mohr, Mrs. E.sther J.; 1320 East 56th St., Chicago 37, Ill. ('32)

Mohr, George J., M.D.; Institute for Psychoanalysis, 43 E. Ohio St., Chicago 11, Ill. (F) ('26)

Morr, Irma E., Bureau of Attendance, Bd. of Education; 25 Minetta Lane, New York 12 (F) ("40)

Montague, Helen, M.D.; 27 West 9th St., New York (F) ('24) 
Montgomery, Elizabeth T.; 1026 Pine St., Winnetka, IIl. ('41)

Moore, Madeline U.; Community Service Soc., 37-34 82nd St., Jackson Heights, N. Y. (F) ('33)

Moore, T. V., M.D.; St. Anselm's Priory, 14th St. \& S. Dakota Ave., N.E., Washington 17, D. C. (F) ('41)

Moore, William E., M.D.; Child Guidance Center, 312 Locust St., Akron 3, Ohio ('44)

Morris, Donald P., M.D.; Southwestern Med. College, 2211 Oak Lawn Ave., Dallas 4, Texas ('46)

Morris, Woodrow W.; University of Michigan, Bureau of Psychological Serv., Room 100, Rackham Bldg., Ann Arbor, Mich. ('46)

Morsh, Mrs. Edith S.; 5570 Chancellor Blvd., Vancouver, B. C., Canada ('28)

Moulton, BRYANt E., M.D.; 18 Symmes Road, Winchester, Mass. ('31)

Moulton, Margaret, Bur. Mental Hygiene, Dept. of Health; 56 Garden St., Hartford 5, Conn. ('46)

Mountain, Johanna C. G., Social Service, Lenox Hill Hospital; 423 W. 120th St., New York 27 ('42)

Moyle, Henry B., M.D.; Hartley-Salmon Clinic, 79 Farmington Ave., Hartford 5, Conn. (F) ('29)

Murphey, Bradrord J., M.D., Univ. of Colorado, College of Medicine; 814 Republic Bldg., Denver 2, Colo. (F) ('29)

Murray, Willa L.; P.O. Box 404, Hilo, T. H. ('30)

Nassar, Carlos, M.D.; Casilla 9319, Santiago, Chile, S.A. ('46)

Nelson, William, M.D.; 5449 Delmar Blyd., St. Louis, Mo. ('29)

Ness, Claire M., M.D., Bur. Men. Hyg., State Dept. Health; 56 Garden St., Hartford 5, Conn. ('44)

Neumann, Frederika; Jewish Board of Guardians, 228 East 19th St., New York 3 (F) ('31)

Newell, H. Whitman, M.D.; Mental Hygiene Clinic, 601 W. Lombard St., Baltimore 1, Md. (F) ('30)

Nicholson, Marian B.; 855 S. Detroit St., Los Angeles 36, Calif. ('40)

Nicolls, Mary M., San Diego City Schools; 3560 4th Ave., San Diego 3, Calif. ('42)

Oberndorf, Clarence P., M.D.; 40 West 59th St., New York 19 (F) ('31)

O'Brien, Frank J., M.D.; Board of Education, 110 Livingston St., Brooklyn, N. Y. (F) ('25)

Olson, Elma; Ment. Hyg. Assoc. Westchester Co., County Office Bldg., White Plains, N. Y. (F) ('29)

Olson, Willard C., Ph.D.; School of Education, University of Michigan, Ann Arbor, Mich (F) ('29)

O’Neili, Mrs. Claire H.; 910 Sir Francis Drake Blvd., San Anselmo, Calif. ("43)

Orgel, Samuel Z., M.D.; 667 Madison Ave., New York 21 (F) ('29)

OrR, Douglass W., M.D.; 4533 East Laurel Drive, Seattle 5, Wash. ('42)

O'Shea, Harriet E., Ph.D.; Purdue University, Psychology Dept., Lafayette, Ind. (F) ('39)

Parker, Z. Rita, M.D.; 115 East 61st St., New York 21 ('28)

Patey, Henry C.; West Riding, Wellesley 81, Mass. ('40)

Patry, Frederick L., M.D.; 218 State St., Albany 6, N. Y. (F) ('32)

Paulsen, Alma A., Bureau Child Guidance; 416 West 20th St., New York 11 ('42)

Paynter, Richard H., Ph.D., Long Island University; 395 Grand Ave., Brooklyn 16, N. Y. (F) ('30)

Pearson, Gerald H. J., M.D., Temple Univ. Med. School; 111 N. 49th St., Phila. 39, Pa. (F) ('30)

Peppard, S. Harcourt, M.D.; Bureau Child Guidance, 228 E. 57 th St., New York 22 (F) ('31)

Perkins, Nellie L., Ph.D.; 208 Delaware Ave. W., Urbana, Ill. ('25)

Perry, Doris E.; 137 Scamman St., So. Portland, Me. ('30)

Polley, Jeannette M.; 28 Scott St., Canfield, Ohio ('32)

Portser, Elinor G.; 423 East Wister St., Germantown, Philadelphia 44, Pa. ('40)

Prather, Mrs. Helen G.; 828 Taylor St., San Francisco 2, Calif. ('43)

Pratt, George K., M.D., Conn. Society for Mental Hygiene; 80 Myrtle Ave., Westport, Conn. (F) ('27)

Preston, Grorge H., M.D.; Bd. Mental Hygiene, 330 N, Charles St., Baltimore 1, Md. (F) ('29)

Pritchett, Henry L., Ph.D.; Box 503, Southern Methodist Univ., Dallas 5, Texas ('31)

Putnam, Marian C., M.D.; Children's Center, 244 Townsend St., Roxbury 21, Mass. (F) ('32)

Quinlan, Gertrude; Catholic Charities Guidance Inst., 133 E. 58th St., New York 22 (F) ('35)

Rabin, Albert I., Ph.D., State Hospital Clinics; 105 Pleasant St. Concord, N. H. ('44)

Rademacher, E. S., M.D.; 442 Temple St., New Haven 11, Conn. (F) ('27)

Rado, Sandor, M.D.; 25 East 86th St., New York 28 (F) ("42)

Ranck, Katherine H.; UNRRA, Foo Chong Hotel, 57 Chung Shan Lu, Nanking, China ('39)

Rank, BeAta; Judge Baker Guidance Center, 38 Beacon St., Boston 8, Mass. (F) ('41) 
Rapaport, David, Ph.D.; The Menninger Clinic, Topeka, Kansas ('44)

Raphael, Theophile, M.D., University of Michigan; 1520 Hill St., Ann Arbor, Mich. (F) ('26)

Rapoport, Jack, M.D., Bellevue Psychiatric Hosp.; 1255 E. 21st St., Brooklyn 30, N. Y. ('43)

Redl, FrItz, Ph.D., Wayne University; 17673 Manderson Drive, Detroit 3, Mich. (F) ('42)

Reeve, George H., M.D., Western Reserve Univ.; 10465 Carnegie Ave., Cleveland 6, Ohio (F) ('26)

Reichard. Suzanne K.; 1075 Cragmont Ave., Berkeley 8, Calif. ('44)

Reichenberg-Hackett, Wally, Ph.D.; Riverdale Country School, Riverdale-on-Hudson, New York 63 (F) ('38)

Reiman, M. Gertrude, Ph.D.; Child Center, Catholic University, Washington 17, D. C. ('42)

Reiss, Oscar, M.D., Univ. S. California, Sch. of Med.; 2200 W. 3rd St., Los Angeles, Calif. ('44)

Reynolds, Bertha C.; 3947-48th St., Long Island City, 4 N. Y. (F) ('30)

Reynolds, Chester L., M.D.; Rochester Guidance Center, 31 Gibbs St., Rochester, N. Y. ('40)

Rich, Gilaert J., M.D.; Guidance Clinic, 515 Public Safety Bldg., Milwaukee 3, Wis. (F) ('29)

Richards, T. W., Ph.D.; Comdr. USNR, Official Separation Centre, San Francisco, Calif, ('41)

Richie, Richard F., M.D.; R.D. 1, Gilbertsville, Pa. ('39)

Richter, Helen G., M.D.; 188 Grandview Ave., Hamden 14, Conn. ('43)

Ridenour, Nina, Ph.D.; N.Y.C. Comm. on Mental Hygiene, 105 E. 22nd St., New York 10 (F) ('41)

Rivlin, Harry N., Ph.D.; Queens College, Dept. of Education, Flushing, N. Y. (F) ('39)

Robie, Theodore R., M.D.; 144 Harrison St., East Orange, N. J. ('30)

Robinson, BRuCe B., M.D.; Bureau of Child Guidance, 31 Green St., Newark 2, N. J. (F) ('29)

Robinson, J. Franklin, M.D.; Children's Serv. Center, 335 S. Franklin St., Wilkes Barre, Pa. ('42)

Robinson, Lindsay E., M.D.; Major, MC, c/o 230 Lakeshore Drive, Rouses Point, N. Y. ('37)

Rockwell, Alice J., Ph.D.; Mental Hygiene Society of Maryland, 601 W. Lombard St., Baltimore 1, Md. (F) ('33)

Rogers, Cart R., Ph.D.; 5844 Stony Island Ave, Chićago 37, Ill. (F) ('34)

Roland, Mary C.; Tri-County Child Glidance Center, 820 N. 3rd St., Harrisburg, Pa. ("42)

Romano, Јohn, M.D.; Strong Memorial Hospital, Rochester 7, N. Y. ('41)

Root, June A.; 2543 Woodburn Ave., Cincinnati 6, Ohio (F) ('31)

Rose, John A., M.D.; Child Guidance Clinic, 1711 Fitzwater St., Philadelphia 46, Pa. ('41)

Rosebrook, Wilda M., Ph.D.; Ohio State University, 321 Arps Hall, Columbus 10, Ohio ('40)

Rosenheim, Frederick, M.D.; Judge Baker Guidance Center, 38 Beacon St., Boston 8, Mass. (F) ('39)

Rosenthal, Pauline, M.D , Bellevue Hospital, Psychiatric Div.; 113 E. 19th St., New York 3 ('40)

Rosenthal, Mrs. William H.; Child Guidance Home, 3149 Harvey Ave., Cincinnati 29, Onio ("40)

Rosenzweig, Leonard, M.D.; Dixmont State Hospital, Dixmont, Pa. ('39)

Rosenzweig, Saul, Ph.D.; Western State Psychiatric Hospital, Pittsburgh 13, Pa. (F) ('35)

Ross, Helen, Inst. for Psychoanalysis; 1151 East 56th St., Chicago 37, III. (F) ('39)

Ross, Mabel, M.D.; Guidance Center, 88 Goodell St., Buffalo 3, N. Y. ('42)

Ross, Mrs. Mildred M.; 2329 S. Taylor Road, Cleveland Heights 18, Ohio (F) ('37)

Rossmax, Max, M.D.; Allentown State Hospital, Allentown, Pa. ('38)

Roth, William F., JR., M.D.; Comdr., MC, USNR, U. S. Naval Hosp., Charleston, S. C. (F) ('35)

Ruggles, Arthur H., M.D.; Butler Hospital, Providence 6, R. I. (F) ('29)

Russell, John A., M.D., Long Beach Ment. Hyg. Clinic; 3741 Falcon Ave., Long Beach 7, Calif. ('40)

Sanders, Helen; National Comm. for Mental Hygiene, 1790 Broadway, New York 19 (F) ('42)

Schaefer, Phyllis D., M.D.; 2100 Massachusetts Ave., Washington 8, D. C. ('41)

Schlezinger, Nathan S., M.D., Jefferson Medical College; 255 So. 17th St., Philadelphia, Pa. ('44)

Schneider, Wilmot F., M.D.; Babies \& Children's Hospital, Cleveland 6, Ohio ('40)

Schrag, A. Ralph, M.D.; Provincial Mental Institute, Edmonton, Alberta, Canada ('41)

Schroeder, Dorothy, Conn. Bureau Mental Hygiene; 136 Four Mile Road, W. Hartford, Conn. ('46)

Schroeder, Paul L., M.D.; Institute for Juvenile Research, 907 S. Wolcott Ave., Chicago I2, Ill. (F) ('30)

Schumacher, Henky C., M.D.; Guidance Center, 2525 Euclid Ave., Cleveland 15, Ohio (F) (26)

Schwartz, Lours A., M.D.; 861 Fisher Bldg., Detroit 2, Michigan (F) ('31)

Scoville, Mildred C.; The Commonwealth Fund, 41 East 57th St., New York 22 (F) ('26)

Sears, Robert R., Ph.D., Univ. of Iowa Child Welf. Research; 1124 Dill St., Iowa City, lowa ("45)

Seeberg, Elizabeth, Ph.D.; Norwood \& Valley Schools, 147 Mason St., Cincinnati 19, Ohio ('28)

Selby, Nathaniel E., M.D., Neurological Institute; 133 East 58th St., New York 22 ('41)

Selling, Lowell S., M.D., Recorders Court; 16196 Cherrylawn Ave,, Detroit, Mich. (F) ('30) 
Senior, Rose D., M.D.; 5612 South Kenwood Ave., Chicago 37, Ill. ('35)

Senn, Milton J. E., M.D.; Payne Whitney Clinic, N. Y. Hospital, 525 E. 68th St., New York 21 ("45)

Shakow, David, Ph.D.; Worcester State Hospital, Worcester 4, Mass. (F) ('30)

Shaskan, Donald, M.D.; Guidance Clinic, State Health Department, Seattle 4, Wash. ('43)

Sheldon, Sybil. F.; 235 Marguerita Lane, Pasadena 2, Calif. ('42)

Shirley, Hale F., M.D.; Stanford Univ. Hosp., Clay \& Webster Sts., San Francisco 15, Calif. (F) ('41)

Shirley, Mary M., Ph.D.; Cambridge-Somerville Youth Study, 820 Massachusetts Ave., Cambridge 38, Mass. ('42)

Siegel, Miriam G., Ph.D.; Jewish Board of Guardians, 228 E. 19th St., New York 3 ('44)

Siewers, Albert B., M.D , Board of Education; 713 E. Genessee St., Syracuse 2, N. Y. (F) ('29)

Silveira, Anfbal, M.D.; Hospital de Juguerí, Estação de Jugerí, S.P.R., São Palilo, Brazil, S. A. ('41)

Silverman, Baruch, M.D.; Ment. Hyg. Inst., 531 Pine Ave. W., Montreal, Quebec, Canada (F) ('31)

Sims, Audrey; Guidance Center, 2525 Euclid Ave., Cleveland 15, Ohio ('40)

Slavson, Samuel R.; Jewish Board of Guardians, 228 E. 19th St., New York 3 ('44)

Slawson, John, Ph.D., American Jewish Committee; 19 E. 88th St., New York 28 (F) ('36)

Sloman, Sophie Schroeder, M.D., Inst. Juvenile Research; 221 S. Ridgeland Ave., Oak Park, Ill. ('46)

Sma lievy, Ruth; University of Pittsburgh, 2206 Cathedral of Learning, Pittsburgh 13, Pa. (F) ('36)

Smith, Barry C.; The Commonwealth Fund, 41 East 57th St., New York 22 (H) ('30)

Smith, Groves Blake, M.D.; Beverly Farms, Inc., Godfrey, Ill. (F) ('30)

Solomon, Joseph C., M.D.; Major, MC, Letterman General Hospital, San Francisco, Calif. (F) ('38)

Solomon, Philip, M.D., Comdr., MC, USNR; c/o 56 Egmont St., Brookline 46, Mass. ('41)

Sommer, Conrad, M.D.; 4500 Olive St., St. Louis 8, Mo. (F) ('38)

Spencer, Douglas, Ph.D.; 400 East 59th St., New York 22 ('32)

Spencer, Harvey, M.D.; Austen Riggs Foundation, Stockbridge, Mass. ("41)

Speyer, Helen; 70 East 77 th St., New York 21 (F) ('37)

Spiegei, Herbert X., M.D.; 115-35 Mayfair Rd., Kew Gardens 15, N. Y. ('45)

Spitz, Rene A., M.D.; 1150 Fifth Avenue, New York 28 ('44)

Stavsky, William H., Ph.D.; 288 South River St., Wilkes-Barre, Pa. ('42)

Steiner, Mrs. Lee R.; 114 West 61st St., New York 23 (F) ('38)

Stellern, Mrs. Florence G., Family Service Society; 293 S. Chester, Pasadena 5, Calif. ('43)

Stephenson, Charles W., M.D.; 130 East Avenue, Burlington, Vt. ('32)

Stevenson, George S., M.D.; Nat. Comm. for Mental Hygiene, 1790 Broadway, New York 19 (F) (C)

Stewart, Kathleen K., M.D., Langley-Porter Clinic; 1517 Eighth Ave., San Francisco 22, Calif. ('45)

Stone, Sy вil A.; U. S. Department of Labor, Children's Bureau, Washington 23, D. C. ('45)

Stoy, Edward G., Ph.D.; Route 1, Box 42, San Luis Obispo, Calif. (F) ('31)

Stranahan, G. Marion, M.D.; 1 Gramercy Park, New York 3 (F) ('31)

Strauss, Bernard V., M.D., Kings County Hospital; 1205 Avenue R, Brooklyn 29, N. Y. ('43)

Strecker, Edward A., M.D.; Pennsylvania Hospital, 111 No. 49th St., Philadelphia 39, Pa. (F) ('24)

Struthers, J. N. P., M.D., Huron Valley Children's Center; Forest Plaza, 715 S. Forest Ave., Ann Arbor, Mich. ('43)

Sullivan, Harry Stack, M.D., Wm. Alanson White Foundation; 9003 Bradley Blvd., Bethesda 14, Md. (F) ('30)

Summer, Mary C.; Children's Memorial Clinic, 1001 E. Clay St., Richmond 19, Va. (F) ('26)

Swackhamer, Gladys V., Psychiatric Clinic, Salvation Army; 27 Barrow St., New York ('40)

Swanson, Florence L., M.D.; Spring Apartment Hotel, 5th \& Spring, Seattle 1, Wash. ('42)

Swirt, Sarah H., Community Service Society; 1035 Fifth Ave., New York 28 (F) ('26)

Sylvester, Emmy, M.D.; Michael Reese Hospital, 29th St. \& Ellis Ave., Chicago 16, Ill. (F) ('41)

Symonds, Percival M., Ph.D.; Teachers College, Columbia University, New York 27 (F) ('37)

Szurex, Stanislaus A., M.D., Langley-Porter Clinic; 319 Cherry St., San Francisco 18, Calif. (F) ('40)

Taft, J. Jessie, Ph.D.; Pennsylvania School of Social Work, 2410 Pine St., Phila. 3, Pa. (F) ('25)

TAlbot, Mira, Bureau of Child Guidance; 10 Mitchell Place, New York (F) ('40)

Tallman, Frank F., M.D.; Comm. Mental Diseases, State Office Bldg., Columbus 16, Ohio (F) ('38)

Taussig, Helen P.; 235 East 22nd St., New York 10 (F) ('31)

Teagarden, Florence M., Ph.D., Univ. of Pittsburgh; 726 Gypsy Lane, Pittsburgh 16, Pa. (F) ('37)

Thompson, Lloyd J., M.D.; 66 Wall St., New Haven 11, Conn. (F) ('29)

Thurrotr, John C., M.D.; 29 East 73rd St., New York 21 (F) ('31)

Tiвout, NeLLY H. C., M.D.; Reynier Vinkeleskade 54, Amsterdam, Z, Holland ('40) 
Tiebout Harry M., M.D.; 215 Milbank Ave., Greenwich, Conn. (F) ('28)

Timme, Arthur R., M.D., Pasadena Child Guid. Clinic; 1930 Wilshire Blvd., Los Angeles 5, Calif. ('44) Tolman, Ruth S., Ph.D.; 345 S. Michigan Ave., Pasadena 5, Calif. ('39)

Topping, Ruth, Home for Girls; 5024 Indiana Ave., Chicago, Ill. ('41)

Towle, Charlotte, University of Chicago; 5741 Kenwood Ave, Chicago 37, Ill. (F) ('30)

Tracy, Anna Belle; School Applied Social Science, Western Reserve Univ., 2117 Adelbert Rd., Cleveland 6, Ohio (F) ('30)

TRUITt, RalPh P., M.D., Ment. Hyg. Soc. of Maryland; 1014 St. Paul St., Baltimore 2, Md. (F) ('30)

Tulchin, Simon H., Smith College School for Social Work; 136 E. 57 th St., New York 22(F) ('28)

Turitz, Mrs. Lillian L., Foster Home Bureau; 310 East 75th St., New York 21 ('45)

Uhler, Claude, M.D.; 223 N. Willomet St., Dallas 8, Texas ('39)

Ulrich, CARL F., M.D., Western Res. Univ. Sch. of Med.; 1615 Hazel Dr., Cleveland 6, Ohio (F) ('30)

Valentine, Ruth, Ph.D.; USPHS Dispensary, 4th \& D St. S.W., Washington, D. C. (F) ('41)

Vander Veer, Adrian H., M.D.; Bobs Roberts Hospital, 920 E. 59th St., Chicago 37, Ill. (F) ('42)

Van Hyning, Mrs. Mary W.; 3139 Oliver St., N.W., Washington, D. C. ('30)

Vedder, Mrs. Sarah, N. Y. Church Mission of Help; Rock Rimmon Road, Stamford, Conn. (31)

Vosk, Mrs. Jeannette S., Bureau of Child Guidance; 167 E. 95th St., New York 28 ('42)

Wadsworth, Morton L., M.D.; 5445 Netherlands Ave., New York 63 ('41)

Waelder, Robert, Ph.D., Bryn Mawr College; 2100 Walnut St., Philadelphia, Pa. ('44)

Wannamaker, Claudia; Inst. for Juvenile Research, 907 S. Wolcott Ave., Chicago 12, Ill. (F) ('41)

Waterman, John H., M.D.; Bureau Mental Hygiene, 304 Mcllhenny St., Houston 6, Texas (F) ('42)

Watson, Goodwin, Ph.D.; Teachers College, Columbia Univ., 525 W. 120th St., New York 27 (F)('33)

Weatherly, Howard E., M.D.; Inst. Junvenile Research, 907 S. Wolcott St., Chicago 12, IIl. ('41)

Webr, Mrs. Evelyn R.; The Guidance Clinic, 129 E. Orange St., Lancaster, Pa. ('45)

Webber, Alice J.; 221 West 82nd St., New York 24 (F) ('31)

Wechsler, David, Ph.D., Bellevue Hosp., Psychiatric Div.; 145 E. 92nd St., New York 28 (F) ('37)

Weider, Arthur, Ph.D.; Medical Division, Caterpillar Tractor Co., Peoria 8, Ill. ('46)

Weill, Blanche C., Ed.D.; 470 West 24th St., New York 11 ('35)

Weiss, Theodore S., M.D., Bellevue Hosp., Psychiatric Div.; 27 W. 96th St., New York 25 ('41)

Wells, Frederic L., Ph.D., Harvard University; 13 Holyoke St., Cambridge 38, Mass. (F) ('39)

Welsch, Exie E., M.D.; Bureau Child Guidance, 193 W. 139th St., New York 20 (F) ('39)

Westburgh Edward M., Ph.D.; The Institute, 111 North 49th St., Philadelphia 39, Pa. ('39)

Westlund, Norman, M.D., Saginaw Valley Chn's Center; 122 S. Charles St., Saginaw, Mich. (F) ('41)

Whitaker, CArl, M.D.; Oak Ridge Hospital, Oak Ridge, Tenn. ('42)

White, Blanche H.; 3622 Park, Kansas City, Mo. ("40)

Wickman, E. K.; The Commonwealth Fund, 41 E. 57 th St., New York 22 (F) ('28)

Wickman, Katharine M.; Pediatric Psychiatric Clinic, Babies Hosp., 167 th \& Broadway, New York 32 (F) ('29)

Wilderson, Myrna; Park Lane, Chagrin Falls, Ohio ('37)

Winters, Alfred G.; Children's Aid Society, 10427 Detroit Ave., Cleveland, Ohio ('42)

Winters, Ruth R.; Children's Aid Society, 10427 Detroit Ave., Cleveland, Ohio ('42)

Wrtkin, Helen, Nat'l Council Jewish Women; UNRRA Team 552, c/o APO 757, PM, N. Y. ('46)

Witmer, Helen L., Ph.D., Smith College Sch. Soc. Work; 308 Quaker Lane, Alexandria, Va. (F) ('34)

Witmer, Lightner; Devon, Pa. ('25)

Wittengerg, Rudol.Ph M., Ph.D., Bureau Child Guidance; 50 E. 89th St., New York 28 ('45)

Witrenbrook, John M., M.D.; Guidance Center, 2525 Euclid Ave., Cleveland 15, Ohio ('44)

Wolrf, ERNst, M.D.; 2535 Vallejo St., San Francisco 23, Calif. (F) ('39)

Wollan, Kenneth I., S.Sc.D.; Connecticut Jr. Republic, Litchfield, Conn. ('40)

Woltmann, Adolf G.; 115 East 116th St., New York 29 (F) ('39)

Wood, Lovis ; Counseling Department Y.W.C.A., 140 Clarendon St., Boston, Mass. (F) ('38)

Woods, Mrs. Lawrence C., Jr.; 270 Grant St., Sewickley, Pa. (F) ('39)

Woodward, Luther E., Ph.D., Nat. Comm. Ment. Hyg.; 2400 Avenue M., Brooklyn 10, N. Y. (F) ('40)

Woolf, Henriette, Bureau of Child Guidance; 237 West 15 St., New York 11 ('42)

Woolley, Lawrence F., M.D.; 1607 Barclay Place, N.E., Lenox Park, Atlanta, Ga. (F) ('32) 
Wortis, JosePh, M.D.; 152 Hicks St., Brooklyn 2, N. Y. ('43)

Wyatt, Frederick, Ph.D., McLean Hospital; 17 Wright St., Cambridge 38, Mass. ('45)

Xelowsri, Mary Louise; The Guidance Center, 1737 Prytania Ave., New Orleans 13, La. ('46)

Yarnell, Helen, M.D.; Mental Hygiene Clinic, 111 E. Front St., Plainfield, N. J. ('40)

Young, Paul C., Ph.D.; Louisiana State University, Dept. Psychology, Baton Rouge 3, La. ('37)

Young, Robert A., Ed.D., Judge Baker Guid. Center; 17 Oak Knoll, Arlington 74, Mass. (F) ('40)

Zeitlin, Goldie R., Bureau Child Guidance; 78-14 138th St., Kew Garden Hills, L. 1., N. Y. ('41) Zilboorg, Gregory, M.D., Psychoanalytic Institute; 14 E. 75th St., New York 21 (F) ('32)

Zimmerman, Kent A., M.D.; U. S. Dept. of Labor, Chilảren's Bureau, Washington 25, D. C. ('46) 